\title{
Square-wave switching in vertical-cavity surface-emitting lasers with polarization-rotated optical feedback: Experiments and simulations
}

\author{
David W. Sukow, ${ }^{1}$ Taylor Gilfillan, ${ }^{1}$ Brenton Pope, ${ }^{1}$ Maria S. Torre, ${ }^{2}$ Athanasios Gavrielides, ${ }^{3}$ and Cristina Masoller ${ }^{4}$ \\ ${ }^{1}$ Department of Physics and Engineering, Washington and Lee University, Lexington, Virginia 24450, USA \\ ${ }^{2}$ Instituto de Física 'Arroyo Seco', UNCPBA, Pinto 399 (7000) Tandil, Argentina \\ ${ }^{3}$ Air Force Research Laboratory AFRL/EOARD, 86 Blenheim Crescent, Ruislip Middlesex HA4 7HB, United Kingdom \\ ${ }^{4}$ Departament de Física i Enginyeria Nuclear, Universitat Politècnica de Catalunya, Colom 11, 08222 Terrassa, Barcelona, Spain
}

(Received 27 March 2012; revised manuscript received 24 July 2012; published 12 September 2012)

\begin{abstract}
We study experimentally the dynamics of vertical-cavity surface-emitting lasers (VCSELs) with polarizationrotated (PR) optical feedback, such that the natural lasing polarization of a VCSEL is rotated by 90 deg and then is reinjected into the laser. We observe noisy, square-wave-like polarization switchings with periodicity slightly longer than twice the delay time, which degrade to (or alternate with) bursts of irregular oscillations. We present results of simulations that are in good agreement with the observations. The simulations demonstrate that close to threshold the regular switching is very sensitive to noise, while well above threshold is less affected by the noise strength. The frequency splitting between the two polarizations plays a key role in the switching regularity, and we identify wide parameter regions where deterministic and robust switching can be observed.
\end{abstract}

DOI: 10.1103/PhysRevA.86.033818

PACS number(s): 42.55.Px, 42.60.Mi, 42.65.Pc

\section{INTRODUCTION}

Optical feedback-induced phenomena in semiconductor lasers (SLs) is an active research field that attracts a lot of attention, motivated, on one hand, by the many applications of external-cavity SLs and, on the other hand, from a nonlinear science point of view, for the rich variety of complex behaviors that are induced by the time-delayed feedback [1-4].

An optical feedback scheme that has received a lot of attention has been referred to as polarization-rotated (PR) feedback or orthogonal feedback [5-14]. In this scheme a 90-deg polarization-rotating device is placed in the external cavity, and thus, the associated linear orthogonal polarizations (in the following referred to as $x$ and $y$ ) are mutually fed back: $x$-polarized light is reinjected into the laser as $y$-polarized light after a delay time $\tau$, and vice versa. This feedback scheme has attracted attention because it can generate all-optically square-wave (SW) switching, with a periodicity slightly longer than $2 \tau$.

Several experiments have been performed in which only one polarization, the natural lasing one, is selected and fed back into the orthogonal one [15-18]. With this scheme, referred to as selective orthogonal feedback, it has been observed that the feedback linearly shifts the laser emission frequency $[15,16]$ and, under strong feedback, polarization SWs have also been observed $[17,18]$. Simulations based on the spin-flip model for vertical-cavity surface-emitting lasers (VCSELs) [17] and on a two-mode model for edge-emitting lasers (EELs) [18] showed a good agreement with the observations. More complex wave forms were also observed, both experimentally and numerically.

High-frequency, regular SWs are interesting for many applications and they have been studied in other optoelectronic systems [19]. Selective orthogonal coupling, such that the dominant mode of one laser is rotated by $90 \mathrm{deg}$ and then injected into the naturally suppressed mode of another laser (and vice versa for mutual coupling), is also capable of producing all-optically square-wave switching [20]. With delays that are of a few nanoseconds, a main advantage of the feedback or coupling schemes is that they are capable of producing SWs with a repetition rate in the gigahertz range, that is tuned by the delay time of the feedback or of the coupling.

In this article we study experimentally and numerically SWs in VCSELs induced by selective orthogonal feedback. VCSELs are nowadays widely employed in photonics applications as they have many advantages as compared to EELs [21]. A first goal of our work is to compare SWs in VCSELs with those in EELs [18]. Since VCSELs present a polarization behavior that strongly differs from that of EELs, it can be expected that the SWs in these lasers will present different features. For example, a few of us recently showed numerically [22] that in VCSELs with selective orthogonal coupling the square waves are more irregular than those in EELs with selective orthogonal coupling [23,24]. Moreover, while in $[23,24]$ the SWs were found to be stable in narrow parameter regions, in [22] they were just a transient dynamic.

A second goal of our work is to find parameters that optimize the regularity of the switchings. Our study extends the work of Mulet et al. [17] that studied experimentally and numerically a VCSEL with orthogonal feedback. In [17] the laser was pumped close to threshold and the simulations were done with parameters that fitted the experimental situation. Here, the experiments are done with VCSELs biased well above threshold, and in the simulations, a wide region of parameters is explored. In particular, we study two types of feedback: when the high-frequency polarization (referred to as $y$ ) is injected into the low-frequency one (referred to as $x$ ), and when the low-frequency polarization is injected into the high-frequency one. These two feedback types will be referred to as $y \rightarrow x$ and $x \rightarrow y$, respectively. In [17] the laser parameters that fitted the experimental situation corresponded to $y \rightarrow x$ feedback.

Experimentally, we observe square-wave-like polarization switchings that degrade to (or alternate with) bursts of irregular and faster oscillations, and the simulations are found to be in very good agreement with the observations. The simulations 
also indicate that close to threshold the regularity of the switchings is very sensitive to noise, while well above the threshold is less affected by the noise strength. The frequency splitting between the two polarizations plays a key role in the switching regularity, and we identify wide parameter regions where deterministic and robust switching can be observed.

This article is organized as follows. The experimental setup and observations are presented in Sec. II. Section III presents the spin-flip rate equation model often employed to describe the polarization dynamics of VCSELs, extended to account for polarization-rotated time-delayed feedback. Section IV presents the results of the simulations, where we find model parameters (pump current, birefringence) for which the switching regularity is optimal and most robust to noise. Our conclusions are presented in Sec. V.

\section{EXPERIMENT}

In our experiments we employ a VCSEL fabricated to operate in a single longitudinal mode and single transverse mode (Finisar SV3639-001, $\lambda=856 \mathrm{~nm}$ when stabilized in temperature at $18.00{ }^{\circ} \mathrm{C}$, the threshold current $I_{\text {th }}=1.18 \mathrm{~mA}$ ). The stand-alone VCSEL (in the absence of feedback) does not display polarization switching or polarization instabilities.

The experimental setup is shown in Fig. 1. The dynamical system with selective orthogonal optical feedback is formed by a linear external cavity of length $153 \mathrm{~cm}$ and photon round-trip time of $\tau=10.2 \mathrm{~ns}$. The laser (LD) emission is collimated by a lens (CL, Newport F-L20, numerical aperture 0.50$)$. A half-wave plate $(\lambda / 2)$ compensates for the initially non-Cartesian orientation of the polarization, thus allowing for more accurate $90^{\circ}$ feedback rotation and better polarization-resolved detection. The beam then passes through a nonpolarizing plate beamsplitter (BS) that transmits $70 \%$ of the incident power, and continues through a Faraday rotator (ROT), made of a Faraday isolator with the input polarizer removed and output polarizer oriented $45^{\circ}$ from horizontal. The beam, now linearly polarized at $45^{\circ}$, passes through a rotatable polarizer (POL) used to control feedback strength, then reflects from a high-reflectivity mirror (HR). On the return path, the beam first passes unchanged through the rotatable polarizer and then reenters the Faraday through its output polarizer. The beam, again forced into $\mathrm{a} 45^{\circ}$ linear polarization, now rotates to $90^{\circ}$, and finally passes through the $\lambda / 2$ plate so it

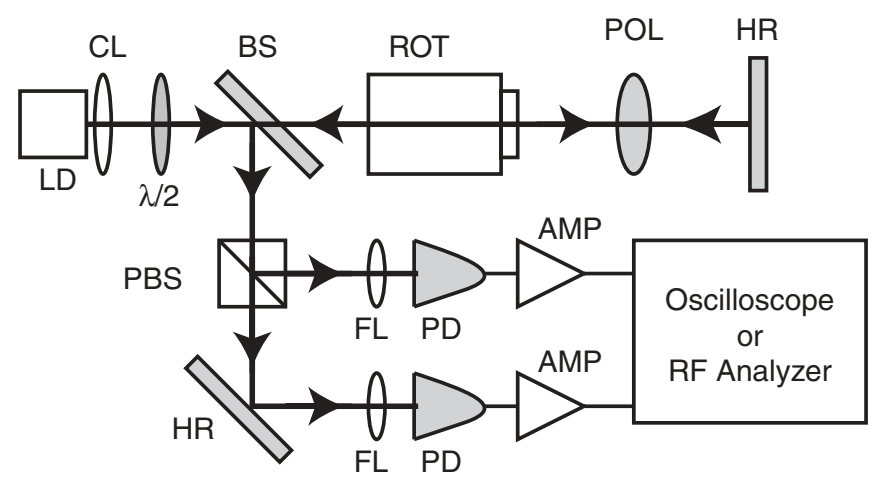

FIG. 1. Experimental schematic diagram. Abbreviations are defined in text. is reinjected into the VCSEL in a polarization state orthogonal to the natural mode of emission. This configuration creates polarization-selective feedback, since vertically polarized light is extinguished by the Faraday rotator. This also assures the feedback arises from only a single round trip in the external cavity.

Polarization-resolved detection is needed to observe the dynamical effects most clearly. The reflected beam from the plate beamsplitter BS is steered to a polarizing beamsplitter cube (PBS). Each of the two resulting beams passes through a focusing lens (FL) onto an ac-coupled photodetector (PD, Hamamatsu C4258-01, 8.75 GHz bandwidth). A wideband amplifier (AMP, $10 \mathrm{kHz}$ to $12 \mathrm{GHz}, 23-\mathrm{dB}$ gain) strengthens each signal, which then is captured and analyzed with a digital storage oscilloscope (LeCroy 8600, 6-GHz analog bandwidth) or microwave spectrum analyzer (Agilent E4405B).

Experimental results demonstrate that self-modulated square waves produced by the VCSEL in this configuration tend to be noisy and disordered, but can be optimized by the pump current for a given feedback strength. Figure 2 displays time series that illustrate the current dependence of the square waves. For all time series shown in Fig. 2, the delay time $\tau=$ $10.2 \mathrm{~ns}$ and the round-trip power transmission is $30.6 \%$. Each graph shows both polarization modes, captured simultaneously and deskewed to compensate for the 0.45-ns difference in detection path length. The horizontal polarization, which is the natural lasing mode, is shown in dark gray (red color online) and the vertical polarization (the suppressed mode) in light gray (blue color online). The switching regularity appears to be optimal at $I=2.75 \mathrm{~mA}$ [Fig. 2(c)]. Below this current value, the waves degrade by exhibiting rapid oscillations that disrupt the square-wave plateaus. Above the optimum value,

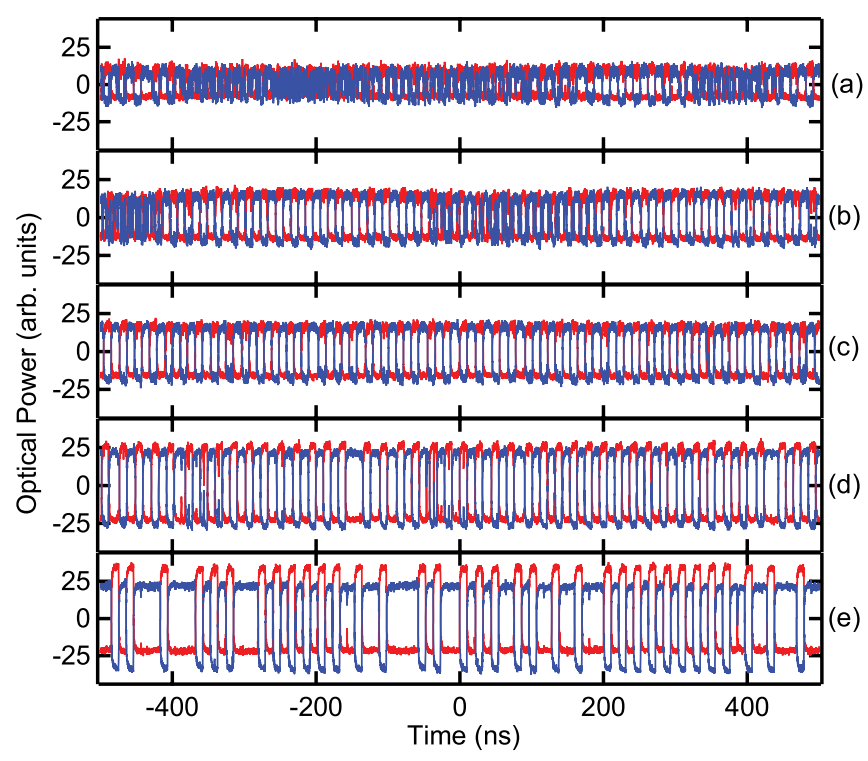

FIG. 2. (Color online) Experimental time series of the intensity of both polarization modes of a VCSEL with PR feedback at various pump currents for fixed feedback strength. Pump currents are (a) $2.20 \mathrm{~mA}$, (b) $2.60 \mathrm{~mA}$, (c) $2.75 \mathrm{~mA}$, (d) $3.20 \mathrm{~mA}$, and (e) $3.40 \mathrm{~mA}$. The horizontal polarization (the natural lasing mode) is shown in dark gray (red color online) and the vertical polarization (the suppressed mode) in light gray (blue color online). 


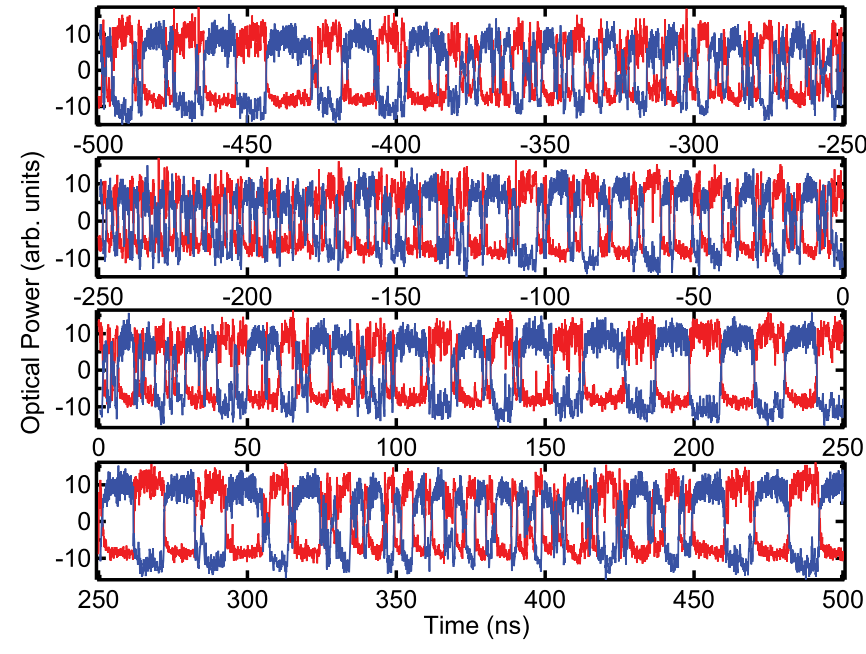

FIG. 3. (Color online) Experimental time series of the intensity of both polarization modes of a VCSEL with PR feedback. External cavity transmission ratio is $30.6 \%$ and pump current $I=2.20 \mathrm{~mA}$. All four graphs are close-up views of the data displayed in Fig. 2(a).

the regularity is also degraded, with longer intervals appearing irregularly between square pulses. For the optimal current the timing of the switchings is not perfectly regular. Other time traces at this setting (not shown) indicate that there are occasional interruptions similar to those in Fig. 2(b), but they occur less often.

Further experiments carried out at other feedback strengths suggest that the optimum current for switching regularity decreases as the feedback strength decreases. However, we also find that the cleanest and most regular square waves occur when feedback is strong.

Figure 3 examines in greater detail the $1-\mu$ s time series shown in Fig. 2(a), with pump current $I=2.20 \mathrm{~mA}$. Different levels of complex behavior are seen, such as almost regular switching, regions where the plateaus become disordered with spikes or holes, and regions of rapid oscillation where the $4 \mathrm{~L} / \mathrm{c}$ periodicity is not apparent. The transitions between these shapes do not appear to be abrupt, and therefore are likely to be a manifestation of noise-driven instability rather than a bifurcation to different solutions.

Similar results (not shown) have been obtained with two other VCSELs, one that displayed polarization monostability without feedback, and the other that exhibited, without feedback, polarization bistability in a range of pump currents. To further demonstrate that the above observations are generic, in the next section we simulate the VCSEL behavior using as a framework the spin-flip model. We employ parameter values that are typically used for modeling VCSEL dynamics, and only the feedback delay time is chosen to fit the experimental value.

\section{RATE EQUATION MODEL}

The spin-flip model equations [25], extended as in Refs. [11, 17] to account for polarization-rotated time-delayed feedback, are:

$$
\frac{d E_{x}}{d t}=k(1+j \alpha)\left[(N-1) E_{x}+j n E_{y}\right]
$$

$$
\begin{aligned}
& -\left(\gamma_{a}+j \gamma_{p}\right) E_{x}+\sqrt{\beta_{s p}} \xi_{x}+\eta_{y} E_{y}(t-\tau), \\
\frac{d E_{y}}{d t}= & k(1+j \alpha)\left[\left(N_{-} 1\right) E_{y}-j n E_{x}\right] \\
& +\left(\gamma_{a}+j \gamma_{p}\right) E_{y}+\sqrt{\beta_{s p}} \xi_{y}+\eta_{x} E_{x}(t-\tau), \\
\frac{d N}{d t}= & \gamma_{n}\left[\mu-N-N I-j n\left(E_{y} E_{x}^{*}-E_{x} E_{y}^{*}\right)\right], \\
\frac{d n}{d t}= & -\gamma_{s} n-\gamma_{n}\left[n I+j N\left(E_{y} E_{x}^{*}-E_{x} E_{y}^{*}\right)\right] .
\end{aligned}
$$

Here, $E_{x}$ and $E_{y}$ are orthogonal linearly polarized field amplitudes, $N$ and $n$ are two carrier densities, $I=\left|E_{x}\right|^{2}+\left|E_{y}\right|^{2}$, $k$ is the field decay rate, $\gamma_{n}$ is the carrier decay rate, $\gamma_{s}$ is the spin-flip rate, $\alpha$ is the linewidth enhancement factor, and $\gamma_{a}$ and $\gamma_{p}$ are the dichroism and birefringence parameters: for $\gamma_{a}>0\left(\gamma_{p}>0\right)$ the $y$ polarization has a lower threshold (a higher frequency) than the $x$ polarization. $\mu$ is the injection current parameter, $\beta_{s p}$ is the strength of spontaneous emission noise, and $\xi_{x, y}$ are uncorrelated Gaussian white noises.

The feedback parameters are the injection strength $\eta$ and the delay time $\tau$. As discussed in the Introduction, we consider two types of selective orthogonal feedback:

(i) $x \rightarrow y$, for which $\eta_{x}=\eta, \eta_{y}=0$ and

(ii) $y \rightarrow x$, for which $\eta_{x}=0, \eta_{y}=\eta$.

Because of the $\alpha$ factor, in the presence of a frequency splitting between the two polarizations, these two types of feedback are not symmetric.

\section{NUMERICAL RESULTS}

Unless otherwise specifically stated, the parameters used in the simulations are $k=300 \mathrm{~ns}^{-1}, \gamma_{n}=2 \mathrm{~ns}^{-1}, \gamma_{s}=50 \mathrm{~ns}^{-1}$,

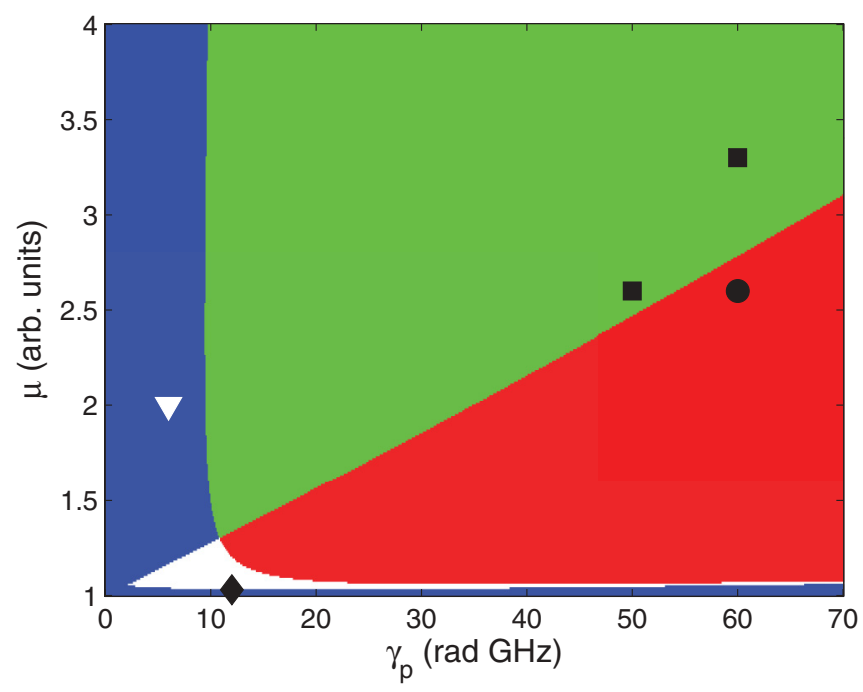

FIG. 4. (Color online) Stability diagram of the $x$ and $y$ polarizations of the solitary VCSEL in the parameter space (birefringence, pump current). Red and blue indicate regions of polarization monostability (red, lower right region: only the $x$ polarization is stable, blue region on the left: only the $y$ polarization is stable); white indicates the region of polarization bistability (both polarizations are stable); green indicates the region where $\mathrm{cw}$ output in $x$ or $y$ polarization is unstable. The model parameters are as indicated in the text, and the symbols indicate the values of $\mu$ and $\gamma_{p}$ used in Figs. 5, 6, and 11. 
$\gamma_{a}=0.4 \mathrm{~ns}^{-1}, \alpha=3, \beta_{s p}=10^{-4} \mathrm{~ns}^{-1}, \eta=50 \mathrm{~ns}^{-1}$, and $\tau=10 \mathrm{~ns}$. The pump current $\mu$ and the birefringence $\gamma_{p}$ are taken as control parameters. Because the experiments were done well above threshold, we will mainly consider values of $\mu \geqslant 2$. However, to compare our results with those of Ref. [17], we will also discuss the dynamics near threshold (with $\mu \sim 1$ ). The parameter $\gamma_{p}$ will be chosen such that the solitary laser is monomode and emits either the $x$ or the $y$ polarization. In Fig. 4, which displays the linear stability of the $x$ and $y$ polarizations, one can observe that well above threshold the $x$ polarization is stable for large $\gamma_{p}$, while the $y$ polarization is stable for low $\gamma_{p}$. Therefore, to analyze the dynamics with $x \rightarrow y$ feedback, we will choose $\gamma_{p}$ large, and to analyze the dynamics with $y \rightarrow x$ feedback, we will choose $\gamma_{p}$ small.

\section{A. Square-wave switching with $\boldsymbol{y} \rightarrow \boldsymbol{x}$ feedback}

The simulations reveal a wide variety of complex wave forms, depending on the parameters. There are parameter regions where almost regular polarization switchings occur, with periodicity slightly longer than $2 \tau$, as shown in Fig. 5. With moderate feedback strength [Fig. 5(a)], the depressed polarization $(x)$ is either off or pulsating, while the natural polarization $(y)$ is either off or constant in time. Figure 5(b) displays a detail of the transition from the $\mathrm{cw}$ to the pulsating state. The period of the pulsations is about $0.09 \mathrm{~ns}$, which is half the relaxation oscillation period $\left(T_{r o}=2 \pi / \sqrt{2 k \gamma_{n}(\mu-1)}=\right.$ $0.18 \mathrm{~ns}$ for the parameters of Fig. 5). Indeed, for lower
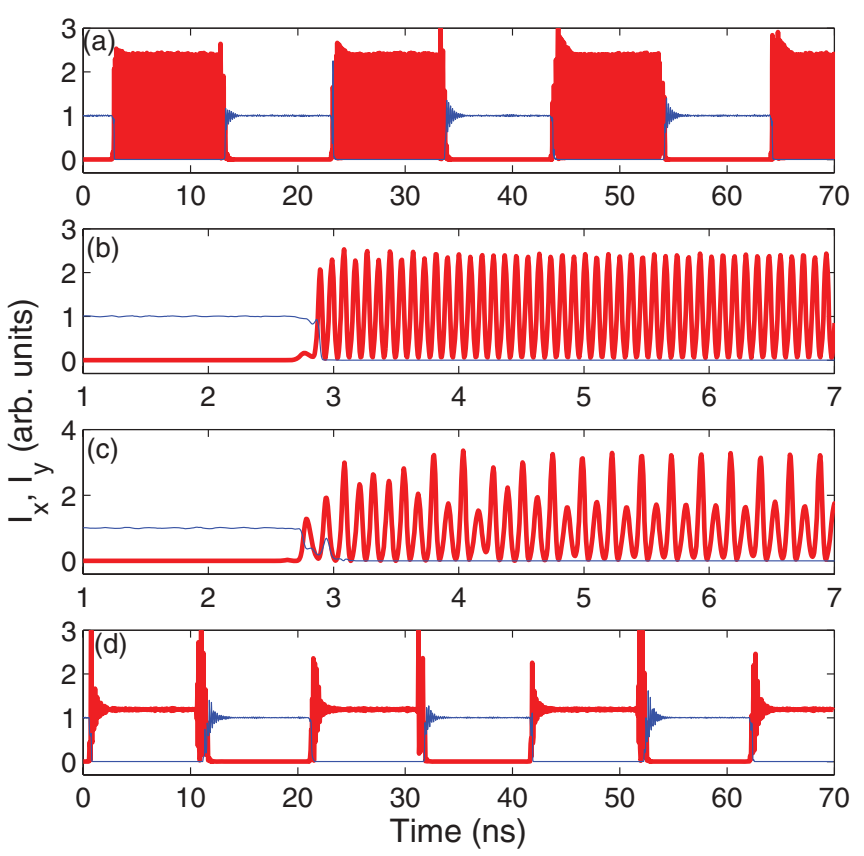

FIG. 5. (Color online) Polarization switching dynamics with $y \rightarrow$ $x$ feedback. The intensities of the $x$ (red, thick line) and $y$ (blue, thin line) polarizations are plotted vs time. In panel (a) the feedback strength is $\eta=50 \mathrm{~ns}^{-1}$; panel (b) displays a detail of the polarization switching in panel (a); panel (c) displays the switching for lower feedback $\left(\eta=28 \mathrm{~ns}^{-1}\right)$ and panel (d), for stronger feedback $(\eta=$ $\left.100 \mathrm{~ns}^{-1}\right)$. The birefringence and pump current parameters are $\gamma_{p}=$ $6 \mathrm{rad} \mathrm{GHz}, \mu=2.0$; other parameters are as indicated in the text. feedback, the pulsations originate from a period-halving bifurcation, as shown in Fig. 5(c). For stronger feedback the pulsations disappear and the intensities of both polarizations display regular square-wave switching, as shown in Fig. 5(d).

Given that the experimental detection bandwidth is 6 $\mathrm{GHz}$, these fast pulsations at frequency twice the relaxation frequency $f_{\text {ro }}$ (with $f_{\text {ro }} \sim 5.5 \mathrm{GHz}$ ) will leave behind only noisy fluctuations observed in the intensity plateaus. These dynamics were observed experimentally in EELs [18], and our simulations suggest that in VCSELs it occurs for $y \rightarrow x$ feedback in a wide range of feedback strengths.

\section{B. Square-wave switching with $x \rightarrow y$ feedback}

For parameters where the $x$ polarization is stable for the solitary laser, with moderate $x \rightarrow y$ feedback strength, regular square-wave switching is observed, as shown in Fig. 6(a), and no fast oscillations have been seen in the depressed $y$ polarization. This difference between $x \rightarrow y$ and $y \rightarrow x$ feedback can be qualitatively understood with the help of an analogy with an optically injected laser. Due to the $\alpha$ factor, the stable locking region is asymmetric with respect to the detuning of the two lasers, which corresponds, for selective orthogonal feedback, to the frequency splitting between the two polarizations. With $y \rightarrow x$ feedback the positive detuning corresponds to parameters in the unstable locking region (resulting in oscillations when the $x$ mode is on), while with $x \rightarrow y$ feedback the detuning changes sign, corresponding to the stable locking region (resulting in a cw intensity when the $y$ mode is on).

For parameters $\left(\gamma_{p}, \mu\right)$ close to the boundary of the region of monostability of the $x$ polarization, in the absence of feedback there is emission in both polarizations, with $I_{y}$ small. The dynamics with $x \rightarrow y$ feedback is displayed in Figs. 6(b) and $6(c)$, where one can observe that the $y$ polarization lases all the time, switching between two intensity plateaus, one higher

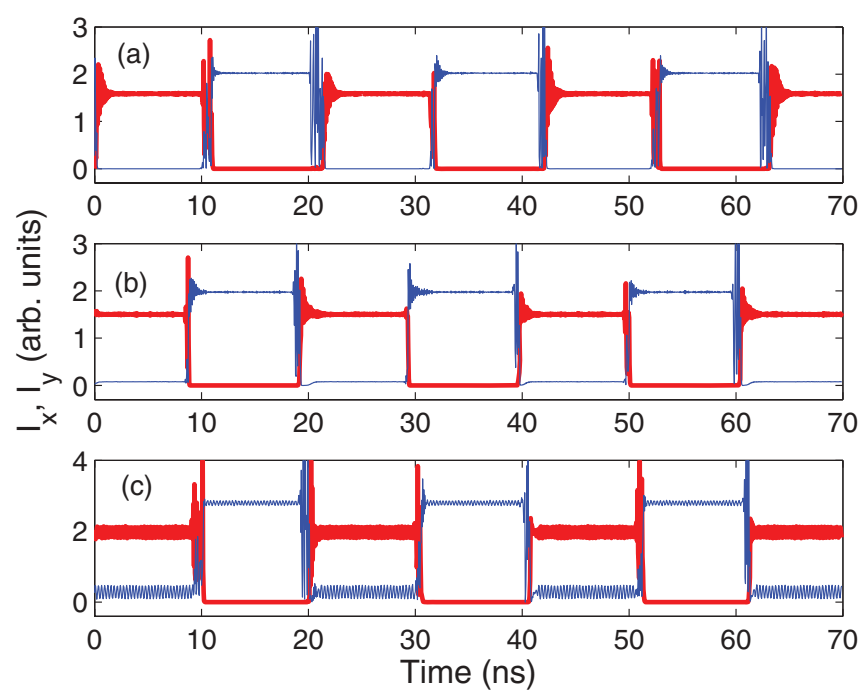

FIG. 6. (Color online) Intensities of the $x$ (red) and $y$ (blue) polarizations with $x \rightarrow y$ feedback. The parameters are $\gamma_{p}=60 \mathrm{rad} \mathrm{GHz}$ and $\mu=2.6$ (a), $\gamma_{p}=50 \mathrm{rad} \mathrm{GHz}$ and $\mu=2.6(\mathrm{~b}), \gamma_{p}=60 \mathrm{rad} \mathrm{GHz}$ and $\mu=3.3$ (c); other parameters are as indicated in the text. 


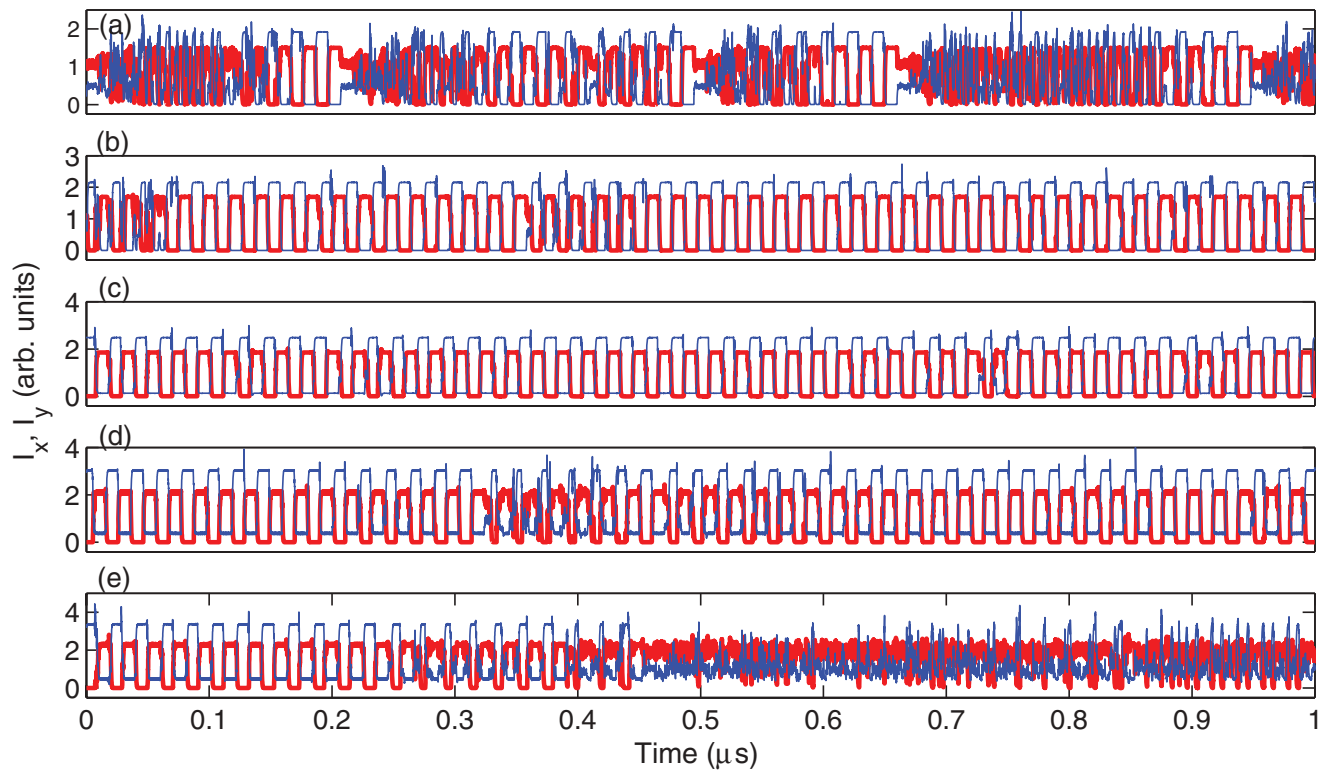

FIG. 7. (Color online) Numerical simulations for $x \rightarrow y$ injection (red line: $x$ polarization, blue line: $y$ polarization). $I_{x}$ and $I_{y}$ were filtered to simulate the 6-GHz experimental bandwidth. $\mu=2.5$ (a), 2.7 (b), 3.0 (c), 3.5 (d), and 3.8 (d), $\eta=50 \mathrm{~ns}^{-1}, \gamma_{p}=60 \mathrm{rad} / \mathrm{ns}$, other parameters are as indicated in the text.

than the solitary laser intensity and one slightly above the noise level.

\section{Characterization of the switching regularity}

For certain parameters a variation of the injection current can affect the regularity of the switchings. This is displayed in Figs. 7 and 8, for $x \rightarrow y$ and $y \rightarrow x$ feedback, respectively. In these figures the intensities were filtered to simulate the 6-GHz experimental bandwidth. There is an optimal range of pump current values where the switching is very regular; for lower $\mu$, intervals of regular switchings alternate with intervals of irregular oscillations, while for higher $\mu$, the switchings degrade to irregular oscillations. One can notice a qualitatively good agreement with the experimental observations (Fig. 2). Unfortunately, as discussed in Sec. V, we could not perform a quantitative comparison because the experimental value of the birefringence parameter could not be measured and the resolution of the detection system did not allow to determine if one polarization lases all the time. However, some differences

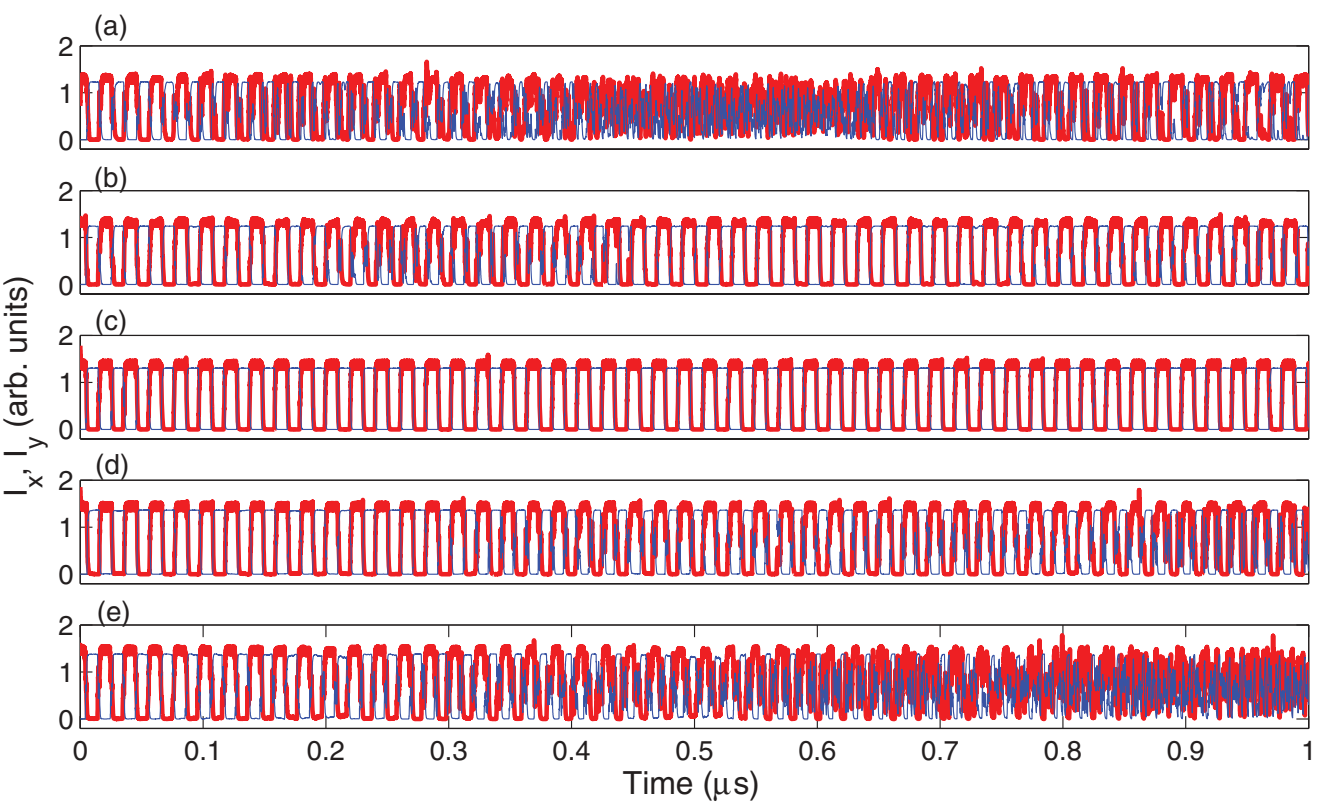

FIG. 8. (Color online) Numerical simulations for $y \rightarrow x$ injection (red line: $x$ polarization, blue line: $y$ polarization). $I_{x}$ and $I_{y}$ were filtered to simulate the 6-GHz experimental bandwidth. $\mu=2.23$ (a), 2.24 (b), 2.3 (c), 2.36 (d), and 2.38 (e), $\eta=23 \mathrm{~ns}^{-1}, \gamma_{p}=6 \mathrm{rad} / \mathrm{ns}$; other parameters are as indicated in the text. 
(a) $\langle T\rangle$ (ns)

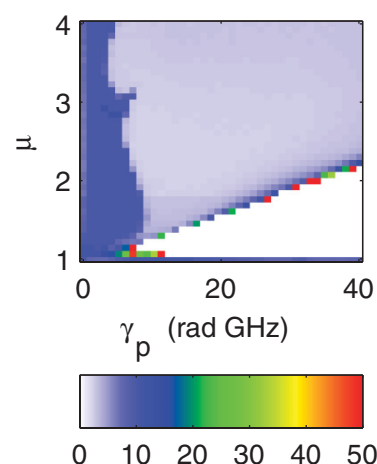

(c) $\langle\mathrm{T}\rangle$ (ns)

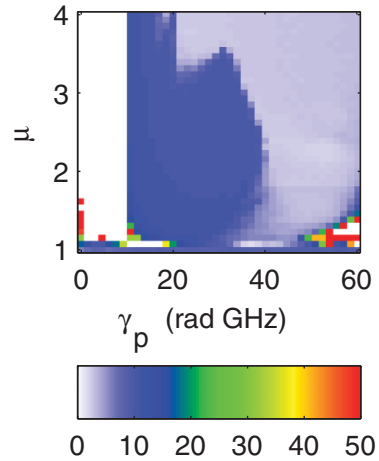

(b) $\sigma /\langle T\rangle$

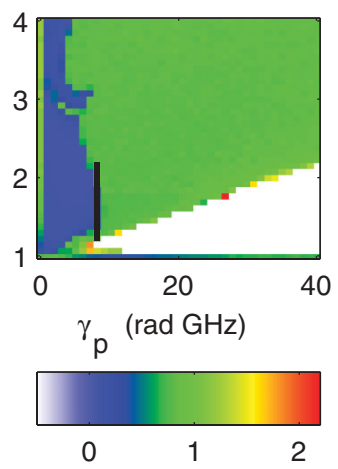

(d) $\sigma /\langle T\rangle$

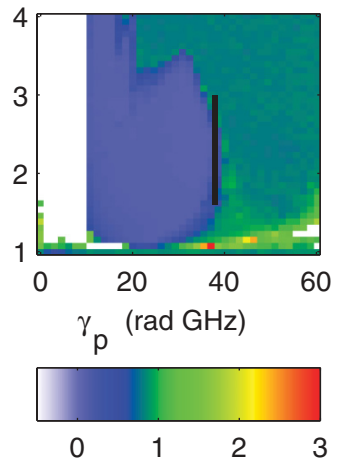

FIG. 9. (Color online) Mean switching time (a), (b) and its normalized standard deviation (c), (d) vs the birefringence and the pump current parameters for $x \rightarrow y$ feedback (a), (c) and for $y \rightarrow x$ feedback (b), (d). The vertical lines in panels (b) and (d) indicate variations of $\mu$ that affect the switching regularity as observed in the experiments. $\eta=30 \mathrm{~ns}^{-1}$; other parameters as indicated in the text. In the while regions no polarization switchings occur; in the red regions, $\langle T\rangle>50 \mathrm{~ns}$.

can indeed be noticed, as in the experiments the amplitude of the square-wave switching appears to be the same for both modes (Fig. 2), while in the simulations they are in general slightly different (see Figs. 5 and 6).

To quantify the degree of switching regularity, in Fig. 9 we plot the average switching time $\langle T\rangle$ and its normalized dispersion $\sigma /\langle T\rangle$ vs $\mu$ and $\gamma_{p}$. In this figure one can observe that there are parameter regions where increasing $\mu$ results in first a decrease, followed by an increase of the switching regularity $(\sigma /\langle T\rangle$ reaching a minimum at specific current values that depend on the value of $\gamma_{p}$ ), as observed in the experiments. For parameters near the borders of the monostability regions of the solitary laser, the mean switching time becomes increasingly long until the switchings eventually disappear [the red color in Figs. 9(a) and 9(c) indicates $\langle T\rangle>$ $50 \mathrm{~ns}]$.

In Ref. [17] the degradation of the switching was understood as the result of a change of stability of the solitary laser polarizations. However, for parameters considered here (i.e., well above threshold) the degradation of the square waves is not related to a change of stability of the solitary laser modes. Specifically, for the parameters of Fig. 7, for the solitary laser the $y$ polarization remains stable as $\mu$ increases [see Fig. 4(a)] and nevertheless, the square-wave regular switchings gradually transform into irregular oscillations.

\section{Influence of spontaneous emission noise}

Our simulations also reveal that spontaneous emission noise can play a crucial role on the degradation of the square-wave switching. Figure 10 displays simulations done for the same parameters as in Figs. 7 and 8, at low and high pump currents, but now with $\beta_{s p} \approx 0$. One can observe that for low pump current, without noise there is regular switching [Figs. 10(a) and $10(\mathrm{c})$ ], while with noise, irregular oscillations alternate with regular switching [Figs. 7(a) and 8(a)]. At higher pump current, without noise [Figs. 10(b) and 10(d)] the switching is more regular than with noise [Figs. 7(e) and 8(e)], but nevertheless, the square waves are sporadically interrupted by a irregular oscillations.

These results suggest that at lower pumps spontaneous emission noise is crucial for the degradation of the square waves, while at higher pumps, not only the spontaneous emission noise but also the deterministic nonlinear dynamics (which plays the role of an "effective" noise as in Ref. [26]) are at the origin of the square-wave degradation.

One can therefore conclude that the role of noise depends on the pump current, and then there could be parameter regions where spontaneous emission noise can enhance the switching regularity. This indeed occurs at pump currents close to threshold and is displayed in Fig. 11, which is done with parameters similar to those used in Ref. [17]. One can observe that the square-wave switching is largely noise-induced, and for each polarization, the time intervals spent in the "on" and "off" states are controlled by the noise strength. These results can be interpreted in terms of noise-induced escape of an unstable fixed point (where $x$ is on and $y$ is off).

\section{CONCLUSIONS AND DISCUSSIONS}

We studied the experimental and numerical dynamics of VCSELs with selective orthogonal feedback and we found that the switching dynamics is noisier than in EELs, but parameters can be found where the switching regularity is optimized. The results of the simulations are in qualitatively good agreement with the observations. The simulations also show that with $x \rightarrow y$ feedback:

(i) there is a wider parameter region where regular switching can be observed (as compared to $y \rightarrow x$ feedback as in [17]) and

(ii) the two polarizations alternate between two cw intensity values; in contrast, with $y \rightarrow x$ feedback the $x$ polarization can display self-pulsations in the "on" state that disappear with stronger feedback.

A quantitative comparison between experiments and simulations is limited by the capabilities of the experimental setup as follows:

(i) The ac coupling of the photodetectors and rf amplifiers removes the cw component of the experimental wave forms. Therefore, we could not determine if one polarization does or does not lase all the time [as observed in the simulations, see Fig. 6(c) and Figs. 7(c)-7(e)]. Tests made with an available low-bandwidth $(125 \mathrm{MHz})$ dc-coupled detector were incon- 


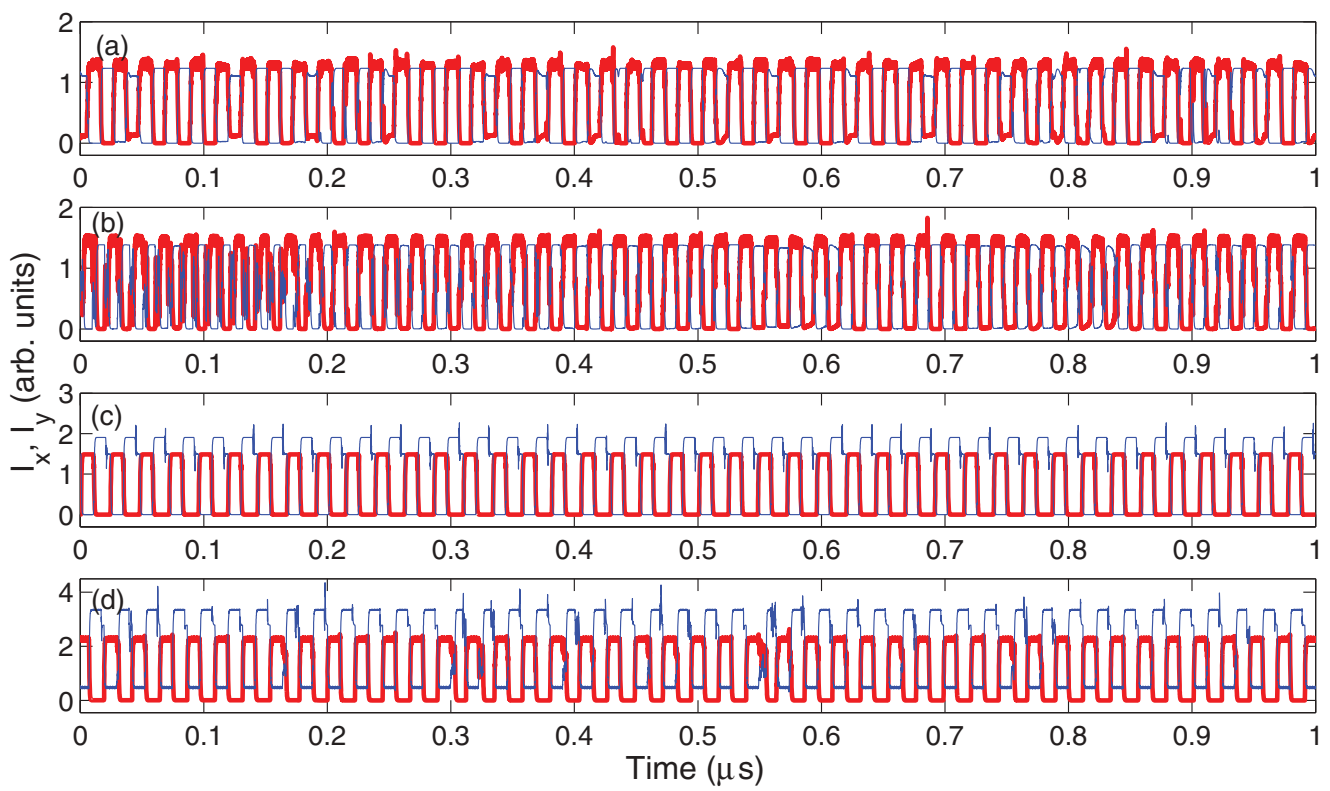

FIG. 10. (Color online) Influence of noise on the degradation of switching regularity. (a), (b) The simulations are done with $\beta_{s p} \approx 0$ and the same parameters as in Figs. 7(a) and 7(e) ( $\mu=2.5$ and $\mu=3.8$, respectively). (c), (d) The simulations are done with $\beta_{s p} \approx 0$ and the same parameters as Figs. 8(a) and 8(e) $(\mu=2.23$ and $\mu=2.38$, respectively).

clusive in this regard; absent further amplification, baseline noise engulfed any small power present.

(ii) The birefringence of the solitary VCSEL could not be measured experimentally because, in the absence of orthogonal feedback, we could not detect the suppressed polarization in the optical spectrum. The VCSEL employed (Finisar SV3639-001) is designed to be single longitudinal and transverse mode (typical SMSR of $30 \mathrm{~dB}$ ). Its spectrum did not display a second mode in all the range of temperature and current studied, within the sensitivity of the optical spectrum analyzer (Newport SuperCavity SR-240-C). The strong suppression of the orthogonal mode could be due to the large dichroism of the laser. At a representative pump current value of the square-wave data, $I=2.99 \mathrm{~mA}$, the extinction ratio $P_{\min } / P_{\max }$ was measured to be $1: 425$. Nearer threshold, at $I=1.50 \mathrm{~mA}$, the ratio was $1: 267$, with greater uncertainty as the minimum power became comparable to the ambient background.

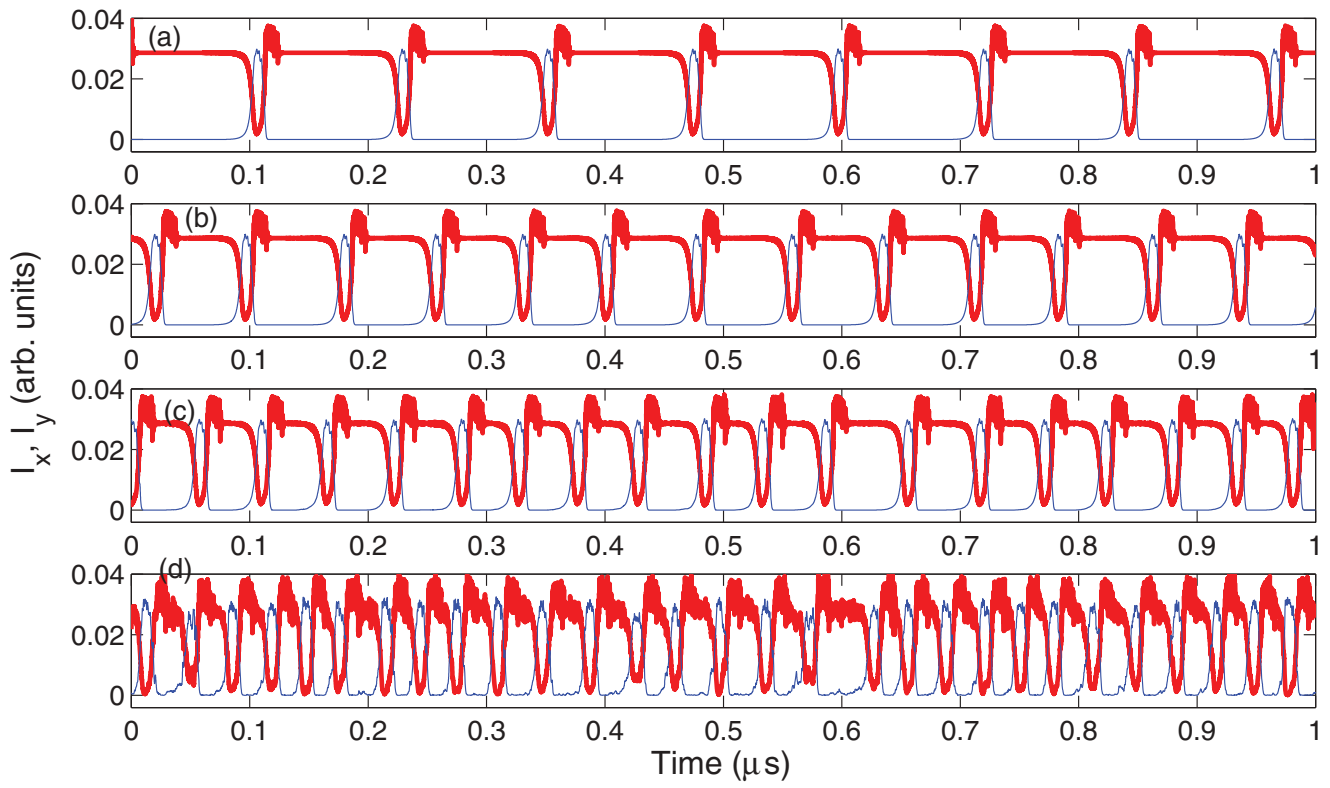

FIG. 11. (Color online) Close to threshold the periodicity of the polarization switchings can be controlled by the noise strength. The parameters are $\mu=1.03$, the $y \rightarrow x$ feedback strength is $\eta=23 \mathrm{~ns}^{-1}, \gamma_{p}=12 \mathrm{rad} / \mathrm{ns}$, and $\beta_{s p}=0$ (a), $10^{-8} \mathrm{~ns}^{-1}$ (b), $10^{-6} \mathrm{~ns}^{-1}$ (c), and $10^{-4} \mathrm{~ns}^{-1}$ (d). 
The simulations also suggest that at high pump currents the switching dynamics is less affected by the noise, as compared to pump current values close to threshold [17]. In addition, at high pump currents the degradation of the regularity of the switchings is not accompanied by a change of the stability of the solitary laser modes (as it occurs at lower pump current [17]), and thus, it will be interesting to investigate in a future work which mechanisms are involved in the degradation of the switching regularity.

\section{ACKNOWLEDGMENTS}

This research was supported in part by the Academia Program of the ICREA foundation, Generalitat de Catalunya; the Air Force Office of Scientific Research through Project FA8655-12-1-2140; the Spanish Ministerio de Ciencia e Innovación through Project FIS2009-13360-C03-02; by the Agència de Gestió d'Ajuts Universitaris i de Recerca, Generalitat de Catalunya, through Project 2009 SGR 1168; and by Argentina CONICET, Grant No. PIP-114-200801-00163.
[1] Unlocking Dynamical Diversity: Optical Feedback Effects on Semiconductor Lasers, edited by D. Kane and A. Shore (Wiley $\&$ Sons, New York, 2005).

[2] J. Ohtsubo, Semiconductor Lasers: Stability, Instability and Chaos, 2nd ed. (Springer, Berlin, 2007).

[3] T. Erneux and P. Glorieux, Laser Dynamics (Cambridge University Press, Cambridge, UK, 2010).

[4] K. Lüdge, Nonlinear Laser Dynamics: From Quantum Dots to Cryptography (Wiley-VCH Verlag GmbH \& Co. KGaA, 2012).

[5] W. H. Loh, Y. Ozeki, and C. L. Tang, Appl. Phys. Lett. 56, 2613 (1990).

[6] S. Jiang, Z. Pan, M. Dagenais, R. A. Morgan, and K. Kojima, Appl. Phys. Lett. 63, 3545 (1993).

[7] F. Robert, P. Besnard, M. L. Charès, and G. Stéphan, IEEE J. Quantum Electron. QE-33, 2231 (1997).

[8] M. Sciamanna, F. Rogister, O. Deparis, P. Megret, M. Blondel, and T. Erneux, Opt. Lett. 27, 261 (2002).

[9] T. Heil, A. Uchida, P. Davis, and T. Aida, Phys. Rev. A 68, 033811 (2003).

[10] D.-L. Cheng, T.-C. Yen, J.-W. Chang, and J.-K. Tsai, Opt. Commun. 222, 363 (2003).

[11] A. Gavrielides, T. Erneux, D. W. Sukow, G. Burner, T. McLachlan, J. Miller, and J. Amonette, Opt. Lett. 31, 2006 (2006).

[12] Y. Takeuchi, R. Shogenji, and J. Ohtsubo, Appl. Phys. Lett. 93, 181105 (2008).

[13] Y. Takeuchi, R. Shogenji, and J. Ohtsubo, Opt. Rev. 17, 144 (2010).
[14] S. Xiang, W. Pan, L. Yan, B. Luo, X. Zou, N. Jiang, and K. Wen, Opt. Lett. 36, 310 (2011).

[15] C. Masoller, T. Sorrentino, M. Chevrollier, and M. Oria, IEEE J. Quantum Electron. 43, 261 (2007).

[16] T. Sorrentino, O. Di Lorenzo, L. C. de Oliveira, M. Chevrollier, and M. Oria, J. Opt. Soc. Am. B. 27, 1458 (2010).

[17] J. Mulet, M. Giudici, J. Javaloyes, and S. Balle, Phys. Rev. A 76, 043801 (2007).

[18] A. Gavrielides, D. W. Sukow, G. Burner, T. McLachlan, J. Miller, and J. Amonette, Phys. Rev. E 81, 056209 (2010).

[19] M. Peil, M. Jacquot, Y. K. Chembo, L. Larger, and T. Erneux, Phys. Rev. E 79, 026208 (2009).

[20] D. W. Sukow, A. Gavrielides, T. Erneux, B. Mooneyham, K. Lee, J. McKay, and J. Davis, Phys. Rev. E 81, 025206(R) (2010).

[21] A. Larsson, IEEE J. Sel. Top. Quantum Electron. 17, 1552 (2011).

[22] M. S. Torre, A. Gavrielides, and C. Masoller, Opt. Express. 19, 20269 (2011).

[23] C. Masoller, D. Sukow, A. Gavrielides, and M. Sciamanna, Phys. Rev. A 84, 023838 (2011).

[24] M. Sciamanna, M. Virte, C. Masoller, and A. Gavrielides, Phys. Rev. E 86, 016218 (2012).

[25] J. Martin-Regalado, F. Prati, M. San Miguel, and N. B. Abraham, IEEE J. Quantum Electron. 33, 765 (1997).

[26] J. F. Martinez Avila, H. L. D. de S. Cavalcante, and J. R. Rios Leite, Phys. Rev. Lett. 93, 144101 (2004). 